\title{
Probability of Disease Extinction or Outbreak in a Stochastic Epidemic Model for West Nile Virus Dynamics in Birds
}

\author{
Milliward Maliyoni
}

Received: date / Accepted: date

\begin{abstract}
Thresholds for disease extinction provide essential information for the prevention and control of diseases. In this paper, a stochastic epidemic model, a continuous-time Markov chain, for the transmission dynamics of West Nile virus in birds is developed based on the assumptions of its analogous deterministic model. The branching process is applied to derive the extinction threshold for the stochastic model and conditions for disease extinction or persistence. The probability of disease extinction computed from the branching process is shown to be in good agreement with the probability approximated from numerical simulations. The disease dynamics of both models are compared to ascertain the effect of demographic stochasticity on West Nile virus dynamics. Analytical and numerical results show differences in model predictions and asymptotic dynamics between stochastic and deterministic models that are crucial for the prevention of disease outbreaks. It is found that there is a high probability of disease extinction if the disease emerges from exposed mosquitoes unlike if it emerges from infectious mosquitoes and birds. Finitetime to disease extinction is estimated using sample paths and it is shown that the epidemic duration is shortest if the disease is introduced by exposed mosquitoes.
\end{abstract}

Keywords Markov chain - West Nile virus · Multitype branching process · Finite-time extinction

Mathematics Subject Classification (2000) 91B70 - 92B05 - 92D25 · 92D30

Milliward Maliyoni

Mathematical Sciences Department, University of Malawi, Chancellor College

P. O. Box 280, Zomba, Malawi

Tel.: +265888353599

E-mail: mmaliyoni@cc.ac.mw

ORCID iD: 0000-0003-4680-6715 


\section{Introduction}

West Nile virus (WNV) is an emerging mosquito-borne arbovirus that belongs to the Flavivirus genus (Bergsman et al. 2016; Mackenzie et al. 2004). WVN can cause fatal neurological diseases by infecting the central nervous system of various host species (Chen et al. 2016; Hayes and Gubler 2006). The transmission cycle of WNV involves wild and domestic birds as primary hosts and mosquitoes, mainly of the Culex genus, as primary vectors of the virus even though the different Culex species responsible for transmission vary greatly by region (Bergsman et al. 2016; Chen et al. 2016; Chevalier et al. 2014). The virus can infect multiple bird species which is a key factor in how it has spread so rapidly worldwide (Bergsman et al. 2016). For instance, WVN was introduced to the Middle East by migrating white storks (Bergsman et al. 2016; Chatterjee et al. 2008). In North America, the first recorded epidemic of WNV was detected in New York State in 1999 and spread rapidly across North America and into Latin America and the Caribbean (Chen et al. 2016; Marfin and Gubler 2001; Wonham and Lewis 2008) presumably after being introduced by migratory birds (Chen et al. 2016). Firstly, the virus was isolated from a dead American crow (Lanciotti et al. 1999) and then from carcasses of many other bird species that were collected between August and November 1999 (Eidson et al. 2001; Steele et al. 2000). The infection in a bird is often the result of bites from infectious mosquitoes in which some species of birds show symptoms of the disease and may die, while other species serve as reservoirs and do not show signs of disease (Chen et al. 2016). According to the US Center for Disease Control (CDC), WNV was found in 326 species of birds.

Under some favourable environmental conditions, the WVN transmission cycle may lead to human and horse infections, which are considered to be dead-end hosts (Chevalier et al. 2014; Gardner et al. 2007). Although the majority of human cases remain asymptomatic (Chevalier et al. 2014), around $30 \%$ of infected people get sick, and show symptoms that range from a flu syndrome to encephalitic diseases, with recent reported case fatality rates ranging from 3\% to 17\% (Ross et al. 2010; Sambri et al. 2013). Further progression of WNV becomes either West Nile meningitis, West Nile encephalitis, or West Nile poliomyelitis with about 1 in every 150 infected people developing severe neurological damage, which can be permanent (Bergsman et al. 2016). The unprecedented level of human, bird, and horse mortality was attributed to a highly virulent emerging strain of the virus (Petersen and Roehrig 2001; Wonham and Lewis 2008). The risk of WNV can be incredibly reduced by using insect repellent and wearing long-sleeved shirts and long pants to prevent mosquito bites (CDC 2012). The CDC introduced the following measures for mosquitoes to prevent and control WNV: reduction of mosquito breeding sites; community outreach and civic education; use of chemical and biological methods to control mosquito adult and larvae; surveillance i.e., monitoring where virus transmission is occurring (CDC 2012).

Several mathematical models have been utilised over the years to help understand, and mitigate the spread of WNV. For instance, Bergsman et al. 
(2016), developed a deterministic model for the transmission of WNV that incorporated resident and migratory birds. Thomas and Urena (2001) employed a difference equation model involving birds, mosquitoes, and humans to investigate the effectiveness of pesticide spraying to reduce mosquito populations and in succession human WNV encephalitis in New York City after the 1999 outbreak. Chen et al. (2016), proposed a deterministic model by including interactions among mosquitoes, birds, and humans to study the local transmission dynamics of WNV. The model was validated by using it to simulate the WNV human data of infected cases and accumulative deaths from 1999 to 2013 in the states of New York, Florida, Texas, and California as reported to the CDC. Bowman et al. (2005) incorporated the human population in infected bird-mosquito models to estimate human cases. Wonham et al. (2004) used a deterministic SIR model of WNV cross-infection between birds and mosquitoes, to provide a method for determining essential mosquito control levels. Wonham and Lewis (2008) described the steps needed to formulate, analyze and apply epidemiological models to vector-borne diseases. Their models focused on WNV, an emerging infectious disease in North America, but first identified in Africa. Laperriere et al. (2011) proposed a model with an environmental temperature that was used to simulate the WNV dynamics of mosquitoes, birds, horses, and humans. The model was then applied to simulate the monthly WNV data of reported bird, equine, and human cases in the Minneapolis metropolitan area (Minnesota). Other mathematical models that were employed to investigate various problems of WNV include Qiu (2011), Wan and Zhu (2010), Simpson et al. (2008), Unnasch et al. (2006), CruzPacheco et al. (2005). Many mathematical models that have been utilised over the years to address various problems on WNV are deterministic, neglecting the possible impact of stochastic effects on disease dynamics. The dynamics of all biological systems are influenced by stochastic (or random) effects and realistically, it is essential that mathematical models that address these systems must include stochastic effects (Ditlevsen and Adeline 2013). In general, biological systems are not only driven by deterministic mechanisms but also by stochastic effects that are inherent in the systems (Ditlevsen and Adeline 2013).

In deterministic epidemic theory, the basic reproduction number, $\mathfrak{R}_{0}$, is a well-known threshold that is used to predict whether a disease will be eliminated or an outbreak will occur. Extinction thresholds exist for the stochastic counterpart of the deterministic model, a continuous-time Markov chain (CTMC) model, where time is continuous and the random variables are discrete (Allend and Lahodny 2012; Lahodny et al. 2015). The extinction thresholds in stochastic models are closely related to the basic reproduction number but depend on the initial number of infectious individuals for each group (Allen and Lahodny 2012; Maliyoni et al. 2017). The stochastic threshold is determined using the theory of branching processes (Allen and van den Driessche 2013; Allen and Lahodny 2012; Lahodny et al. 2015; Maliyoni et al. 2017). The theory of branching process can be applied in conjunction with the deterministic model to provide additional insights about disease extinction (Allen and 
van den Driessche 2013). In 1955, Whittle used the basic reproduction number to derive an expression for approximating the probability of disease extinction for the susceptible-infectious-recovered (SIR) model. If $I(0)=i$ represents the initial number of infectious individuals and $\mathfrak{R}_{0}>1$, then the probability of disease extinction is approximately $\left(1 / \mathfrak{R}_{0}\right)^{i}$. Thus, the probability of a major outbreak is approximated by

$$
1-\left(\frac{1}{\mathfrak{R}_{0}}\right)^{i} .
$$

This result holds for models with one infectious group only (Allen and Lahodny 2012; Lahodny et al. 2015; Maliyoni et al. 2017). For models with multiple infectious groups, the stochastic threshold is determined by the number of individuals within each group and the probability of disease extinction for each group (Allen and Lahodny 2012; Maliyoni et al. 2017). The expected time to disease extinction can be estimated for stochastic models, which is not possible for deterministic models (Allen 2008; Allen and Burgin 2000). One of the most significant differences between deterministic and stochastic models is their asymptotic dynamics. Stochastic solutions converge to the disease-free equilibrium although the corresponding deterministic solution converges to an endemic equilibrium (Allen 2008; Maliyoni et al. 2017). The CTMC model assumes that the variability in the model is due to demographic variability, which is defined as the variability associated with individual dynamics e.g., births or deaths, transmission, recovery (Allen 2017; Kirupaharan and Allen 2004).

To account for demographic random effects that occur in the transmission process of WNV and are vital in determining the probability of disease extinction or a major outbreak, a CTMC model is considered. Thus, the objectives of this investigation are to: (1) develop a CTMC model based on the assumptions of a deterministic model, (2) approximate the probability of disease extinction or a major outbreak for WNV when the disease is introduced by a few infectious birds or mosquitoes, and (3) compare the disease dynamics of the deterministic and CTMC models with respect to disease extinction and outbreak. The deterministic model is a modification of the model (with larval class) by Wonham and Lewis (2008). Unlike their model, the recovered class and demographic parameters, that is, natural birth and death rate are introduced for birds. With demographic stochasticity, it is possible for an infective to recover before transmitting the infection; thus, recovery affects the probability of disease extinction or outbreak and must not be ignored. Also, the larval class for mosquitoes is removed because the goal of this investigation is to account for demographic stochasticity in the transmission dynamics of WNV and larvae do not take part in disease transmission since only adult susceptible mosquitoes get infected as such their impact is negligible in this regard. Also, in the absence of infection, both the bird and mosquito population sizes are variable.

The organisation of this paper is as follows; in Section 2, a WNV deterministic model is introduced and its basic properties are presented. Stability 
conditions of model equilibria are established in terms of the basic reproduction number. In Section 3, a CTMC model is developed based on the deterministic model. The branching process is applied to derive an explicit expression for calculating the probability of disease extinction or a major outbreak when the basic reproduction number is greater than unity. In Section 4, the dynamics of the disease are illustrated using numerical simulations and estimates for the probability of extinction or an outbreak are presented. A comparison of the disease dynamics of the deterministic and CTMC models is carried out. The last section summarises the results and their implications for WNV control and prevention.

\section{Deterministic Model}

\subsection{Model Development}

We present a deterministic model to study the transmission dynamics of WNV, a modification of the model (with larval class) by Wonham and Lewis (2008). Bird and mosquito populations are divided into epidemiological compartments based on WNV status. The total bird and mosquito population sizes are denoted by $N_{b}(t)$ and $N_{m}(t)$, respectively. The subscript $b$ and $m$, respectively, represent the birds and mosquitoes. The bird population is divided into three sub-classes: susceptible $S_{b}(t)$, infectious $I_{b}(t)$ and recovered $R_{b}(t)$, which satisfy $N_{b}(t)=S_{b}(t)+I_{b}(t)+R_{b}(t)$. The mosquito population is divided into susceptible $S_{m}(t)$, exposed $I_{m}(t)$ and infectious $R_{b}(t)$, with the total mosquito population given by $N_{m}(t)=S_{m}(t)+E_{m}(t)+I_{m}(t)$. Birds and mosquitoes enter their respective susceptible classes at a recruitment rate of $\Lambda_{b}$ and $\Lambda_{m}$. The natural death rates of bird and mosquito populations are $\mu_{b}$ and $\mu_{m}$, respectively. Infectious birds recover from WNV at a rate $\alpha$ otherwise they die due to the infection at a rate $\delta$. The progression rate from exposed to infectious class for mosquitoes is $\gamma$. During a subsequent blood meal, a susceptible mosquito may bite an infectious bird and acquires the infection at a rate

$$
\lambda_{m}(t)=\frac{\hat{\beta}_{m} I_{b}(t)}{N_{b}(t)}
$$

with $\hat{\beta}_{m}=\sigma_{b} \beta_{m}$, where $\sigma_{b}$ is the biting rate of mosquitoes on birds and $\beta_{m}$ is the probability of virus transmission per infectious bite from birds to mosquitoes. Similarly, a susceptible bird becomes infectious after being bitten by an infectious mosquito at a rate

$$
\lambda_{b}(t)=\frac{\hat{\beta}_{b} I_{m}(t)}{N_{b}(t)}
$$

with $\hat{\beta}_{b}=\sigma_{b} \beta_{b}$, where $\beta_{b}$ is the probability of virus transmission per infectious bite from mosquitoes to birds. The following assumptions are made for the model: 


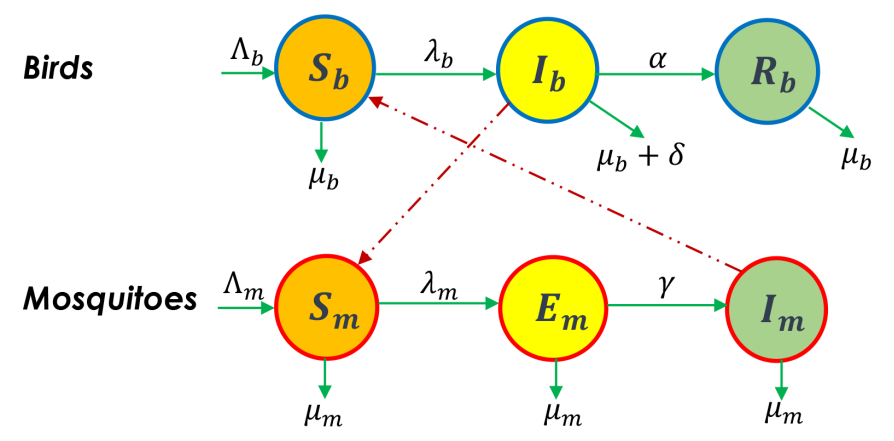

Fig. 1 Compartmental diagram for West Nile virus transmission model

Table 1 Description of state variables for the West Nile virus transmission model

\begin{tabular}{cl}
\hline State variable & Description \\
\hline$S_{b}(t)$ & Number of susceptible birds at time $t$ \\
$I_{b}(t)$ & Number of infectious birds at time $t$ \\
$R_{b}(t)$ & Number of recovered birds at time $t$ \\
$S_{m}(t)$ & Number of susceptible mosquitoes at time $t$ \\
$E_{m}(t)$ & Number of exposed mosquitoes at time $t$ \\
$I_{m}(t)$ & Number of infectious mosquitoes at time $t$ \\
\hline
\end{tabular}

(a) All the recruited birds and mosquitoes are susceptible to the infection and therefore there is no vertical transmission in both the bird and mosquito populations.

(b) Total bird and mosquito populations are variable.

(c) No recovery for infectious mosquitoes because once infected, mosquitoes remain infectious for the remainder of their lifespan (Mpeshe et al. 2011).

(d) Only adult susceptible mosquitoes get infected.

(e) Bites of an infectious mosquito onto an infectious bird are ignored.

(f) Mosquitoes bite the birds randomly i.e., independent of their disease status.

(g) Infectious birds recover with permanent immunity.

The transmission dynamics of the disease are depicted in Figure 1. The state variables and parameters of the model are summarised in Table 1 and 2, respectively.

Based on the assumptions, compartmental diagram 1, state variables (Table 1) and parameters (Table 2), the proposed West Nile virus model satisfies 
Table 2 Description of parameters for the West Nile virus transmission model

\begin{tabular}{cl}
\hline Parameter & Description \\
\hline$\Lambda_{b}$ & Recruitment rate of bird population \\
$\Lambda_{m}$ & Recruitment rate of mosquito population \\
$\beta_{b}$ & Probability of virus transmission per infectious bite from mosquitoes to birds \\
$\beta_{m}$ & Probability of virus transmission per infectious bite from birds to mosquitoes \\
$\sigma_{b}$ & Biting rate of mosquitoes on birds \\
$\mu_{b}$ & Natural death rate of birds \\
$\mu_{m}$ & Natural death rate of mosquitoes \\
$\alpha$ & Recovery rate of infectious birds \\
$\gamma$ & Progression rate of mosquitoes from exposed to infectious class \\
$\delta$ & Disease-induced death rate of infectious birds \\
\hline
\end{tabular}

the following system of nonlinear ordinary differential equations:

$$
\begin{aligned}
\frac{\mathrm{d} S_{b}}{\mathrm{~d} t} & =\Lambda_{b}-\lambda_{b} S_{b}-\mu_{b} S_{b}, \\
\frac{\mathrm{d} I_{b}}{\mathrm{~d} t} & =\lambda_{b} S_{b}-\left(\alpha+\delta+\mu_{b}\right) I_{b}, \\
\frac{\mathrm{d} R_{b}}{\mathrm{~d} t} & =\alpha I_{b}-\mu_{b} R_{b}, \\
\frac{\mathrm{d} S_{m}}{\mathrm{~d} t} & =\Lambda_{m}-\lambda_{m} S_{m}-\mu_{m} S_{m}, \\
\frac{\mathrm{d} E_{m}}{\mathrm{~d} t} & =\lambda_{m} S_{m}-\left(\gamma+\mu_{m}\right) E_{m}, \\
\frac{\mathrm{d} I_{m}}{\mathrm{~d} t} & =\gamma E_{m}-\mu_{m} I_{m} .
\end{aligned}
$$

\subsection{Basic Model Properties}

\subsubsection{Invariant Region}

All the state variables and parameters of model (4) are assumed to be nonnegative for all time $t \geq 0$. Let $\left(S_{b}, I_{b}, R_{b}, S_{m}, E_{m}, I_{m}\right) \in \mathbb{R}^{6}$ be any solution of model (4) with non-negative initial conditions $S_{b}(0), I_{b}(0), R_{b}(0), S_{m}(0), E_{m}(0)$ and $I_{m}(0)$.

The total population of mosquitoes, $N_{m}(t)$, satisfies the differential equation

$$
\frac{\mathrm{d} N_{m}(t)}{\mathrm{d} t}=\Lambda_{m}-\mu_{m} N_{m}(t) .
$$

From equation (5) and the Gronwall inequality, it follows that

$$
N_{m}(t) \leq N_{m}(0) e^{-\mu_{m} t}+\frac{\Lambda_{m}}{\mu_{m}}\left[1-e^{-\mu_{m} t}\right],
$$


where $N_{m}(0)$ represents the initial value of $N_{m}(t)$. Thus, as $t \rightarrow \infty$ we obtain

$$
0 \leq N_{m}(t) \leq \frac{\Lambda_{m}}{\mu_{m}}
$$

Hence, the feasible solutions for the mosquito population enter the domain given by

$$
\Gamma_{m}=\left\{\left(S_{m}, E_{m}, I_{m}\right) \in \mathbb{R}_{+}^{3}: N_{m}(t) \leq \frac{\Lambda_{m}}{\mu_{m}}\right\} .
$$

Likewise, it can be shown using similar approach, that the feasible region for the birds population, $N_{b}(t)$, is given by

$$
\Gamma_{b}=\left\{\left(S_{b}, I_{b}, R_{b}\right) \in \mathbb{R}_{+}^{3}: N_{b}(t) \leq \frac{\Lambda_{b}}{\mu_{b}}\right\} .
$$

Therefore, from equation (6) and (7), the following region

$$
\Gamma=\Gamma_{m} \times \Gamma_{b}
$$

is positively invariant and attracting for the WNV model (4). Thus, it is sufficient to consider the solutions of model (4) in $\Gamma$. For any initial conditions in $\Gamma$, model (4) has unique solutions which start and remain in $\Gamma$ for $t \geq 0$. Furthermore, in the domain $\Gamma$, model (4) is said to be mathematically and epidemiological well-posed (Hethcote 2000).

\subsubsection{Positivity of Solutions}

Theorem 1 Let the initial condition $S_{b}(0)>0, I_{b}(0)>0, R_{b}(0)>0, S_{m}(0)>$ $0, E_{m}(0)>0$ and $I_{m}(0)>0$. So, the solutions $S_{b}(t), I_{b}(t), R_{b}(t), S_{m}(t), E_{m}(t)$ and $I_{m}(t)$ of the $W N V$ model (4) are positive for all $t \geq 0$.

Proof From the first equation of model (4), we have

$$
\frac{\mathrm{d} S_{b}}{\mathrm{~d} t}=\Lambda_{b}-\lambda_{b} S_{b}-\mu_{b} S_{b} \geq-\left(\lambda_{b}+\mu_{b}\right) S_{b} .
$$

Equation (9) can be written as

$$
\frac{\mathrm{d} S_{b}}{S_{b}} \geq-\left(\lambda_{b}+\mu_{b}\right) \mathrm{d} t
$$

Integrating both sides of inequality (10) with respect to $t$ leads to

$$
S_{b}(t) \geq S_{b}(0) e^{-\int_{0}^{t}\left(\lambda_{b}+\mu_{b}\right) d s}>0, \forall t>0 .
$$

Likewise, from the second equation of model (4), we have

$$
\frac{\mathrm{d} I_{b}}{\mathrm{~d} t}=\lambda_{b} S_{b}-\left(\alpha+\delta+\mu_{b}\right) I_{b} \geq-\left(\alpha+\delta+\mu_{b}\right) I_{b}
$$


Integrating (11) with respect to $t$ yields

$$
I_{b}(t) \geq I_{b}(0) e^{-\left(\alpha+\delta+\mu_{b}\right) t}>0, \forall t>0 .
$$

Employing similar approach, it can be shown that $R_{b}(t)>0, S_{m}(t)>$ $0, E_{m}(t)>0$ and $I_{m}(t)>0$ for all $t \geq 0$.

\subsection{Existence and Stability Analysis of Equilibria}

In this section, we investigate the existence and stability of equilibrium points of the WNV model (4).

\subsubsection{Disease-Free Equilibrium}

In the absence of disease in both the bird and mosquito populations, that is, $I_{b}=E_{m}=I_{m}=0$ ), the WNV model (4) has a disease-free equilibrium (DFE) which is given by

$$
\mathcal{E}_{0}=\left(S_{b}^{*}, I_{b}^{*}, R_{b}^{*}, S_{m}^{*}, E_{m}^{*}, I_{m}^{*}\right)=\left(\frac{\Lambda_{b}}{\mu_{b}}, 0,0, \frac{\Lambda_{m}}{\mu_{m}}, 0,0\right) .
$$

The linear stability of $\mathcal{E}_{0}$ is governed by the basic reproduction number, $\mathfrak{R}_{0}$.

\subsubsection{The Basic Reproduction Number}

The basic reproduction number, $\mathfrak{R}_{0}$, is a threshold parameter which is defined as the average number of secondary cases that one typical infected individual can generate if introduced into an entirely susceptible population (Anderson and May 1991; van den Driessche and Watmough 2002; Diekmann et al. 1990). If $\Re_{0}<1$, then on average an infected individual generates less than one new infective over its infectiousness period, and therefore the infection dies out. Conversely, if $\mathfrak{R}_{0}>1$, then each infective averagely produces more than one new infective, and the infection can invade the susceptible population (van den Driessche and Watmough 2002). We derive the expression for $\mathfrak{R}_{0}$ for model (4) using the next generation matrix as described by van den Driessche and Watmough (2002) and Diekmann et al. (1990).

We define $m \times m$ matrices $F$ and $V$, where $m$ represents the number of infected compartments, respectively, as

$$
F=\left[\begin{array}{ccc}
0 & 0 & \hat{\beta}_{b} \\
\frac{\Lambda_{m} \hat{\beta}_{m} \mu_{b}}{\Lambda_{b} \mu_{m}} & 0 & 0 \\
0 & 0 & 0
\end{array}\right] \quad \text { and } \quad V=\left[\begin{array}{ccc}
\alpha+\delta+\mu_{b} & 0 & 0 \\
0 & \gamma+\mu_{m} & 0 \\
0 & -\gamma & \mu_{m}
\end{array}\right]
$$


Thus, the next generation matrix, $F V^{-1}$, is given by

$$
F V^{-1}=\left[\begin{array}{ccc}
0 & \frac{\gamma \hat{\beta}_{b}}{\mu_{m}\left(\gamma+\mu_{m}\right)} & \frac{\hat{\beta}_{b}}{\mu_{m}} \\
\frac{\Lambda_{m} \hat{\beta}_{m} \mu_{b}}{\Lambda_{b} \mu_{m}\left(\alpha+\delta+\mu_{b}\right)} & 0 & 0 \\
0 & 0 & 0
\end{array}\right]
$$

Mathematically, the basic reproduction number is the spectral radius (dominant eigenvalue) of the next generation matrix (14), denoted $\rho\left(F V^{-1}\right)$, and is given by

$$
\mathfrak{R}_{0}=\rho\left(F V^{-1}\right)=\sqrt{\frac{\gamma \hat{\beta}_{b} \bar{\beta}_{m}}{\mu_{m}\left(\gamma+\mu_{m}\right)\left(\alpha+\delta+\mu_{b}\right)}},
$$

where $\bar{\beta}_{m}=\frac{\hat{\beta}_{m} \Lambda_{m} \mu_{b}}{\Lambda_{b} \mu_{m}}$.

The term $\frac{\gamma \hat{\beta}_{b}}{\mu_{m}\left(\gamma+\mu_{m}\right)}\left(\frac{\bar{\beta}_{m}}{\alpha+\delta+\mu_{b}}\right)$ represents the average number of secondary bird (mosquito) infections that one infectious mosquito (bird) generates in a totally susceptible bird (mosquito) population during the entire duration of its infectiousness. The basic reproduction number also provides important information for preventing and controlling the spread of the infection (Mwamtobe et al. 2017).

From Theorem 2 in van den Driessche and Watmough (2002), the following result is established for the local stability of $\mathcal{E}_{0}$.

Theorem 2 The disease-free equilibrium, $\mathcal{E}_{0}$, of the WNV model (4), is locally asymptotically stable in $\Gamma$ if $\mathfrak{R}_{0}<1$, and unstable if $\mathfrak{R}_{0}>1$.

\subsubsection{Endemic Equilibrium}

When there is disease in both the bird and mosquito populations, the WNV model (4) has an endemic equilibrium which is defined by $\varepsilon_{1}=\left(S_{b}^{* *}, I_{b}^{* *}, R_{b}^{* *}, S_{m}^{* *}, E_{m}^{* *}, I_{m}^{* *}\right)$. To determine the condition of existence of $\varepsilon_{1}$, we let

$$
\lambda_{b}^{* *}(t)=\frac{\hat{\beta}_{b} I_{m}^{* *}(t)}{N_{b}^{* *}(t)}
$$

be the force of infection (3) at steady-state. Equating the left side of the equations in model (4) to zero and then solving the resulting equations in terms of $\lambda_{b}^{* *}$, we obtain

$$
\begin{array}{rlrl}
S_{b}^{* *} & =\frac{\Lambda_{b}}{\lambda_{b}^{* *}+\mu_{b}}, & I_{b}^{* *}=\frac{\lambda_{b}^{* *} S_{b}^{* *}}{\alpha+\delta+\mu_{b}}, & R_{b}^{* *}=\frac{\alpha I_{b}^{* *}}{\mu_{b}}, \\
S_{m}^{* *}=\frac{\Lambda_{m}}{\lambda_{m}^{* *}+\mu_{m}}, & E_{m}^{* *}=\frac{\lambda_{m}^{* *} S_{m}^{* *}}{\gamma+\mu_{m}}, & I_{m}^{* *}=\frac{\alpha E_{m}^{* *}}{\mu_{m}} .
\end{array}
$$


Now, substituting the equilibrium values (17) into (16) and then simplifying, satisfy the following quadratic equation (in terms of $\lambda_{b}^{* *}$ ),

$$
\lambda_{b}^{* *}\left(K_{1} \lambda_{b}^{* *}+K_{2}\right)=0
$$

where

$$
\begin{aligned}
& K_{1}=\frac{\Lambda_{b}^{2}\left[\mu_{b} \hat{\beta}_{m}+\mu_{m}\left(\alpha+\delta+\mu_{b}\right)\right]}{\mu_{b}^{2}} \\
& K_{2}=\frac{\Lambda_{b}^{2} \mu_{m}^{2}\left(\gamma+\mu_{m}\right)\left(\alpha+\delta+\mu_{b}\right)-\gamma \mu_{b} \Lambda_{b} \Lambda_{m} \hat{\beta}_{b} \hat{\beta}_{m}}{\mu_{b} \mu_{m}\left(\gamma+\mu_{m}\right)} .
\end{aligned}
$$

Equation (18) has two roots. The first root is $\lambda_{b}^{* *}=0$, and corresponds to the disease-free equilibrium, $\mathcal{E}_{0}$, whose stability has already been ascertained (see Theorem 2). The second root is given by

$$
\lambda_{b}^{* *}=\frac{-K_{2}}{K_{1}}=\frac{\mu_{b} \mu_{m} \Lambda_{b}^{2}\left(\alpha+\delta+\mu_{b}\right)\left(\mathfrak{R}_{0}^{2}-1\right)}{\Lambda_{b}^{2}\left[\mu_{b} \hat{\beta}_{m}+\mu_{m}\left(\alpha+\delta+\mu_{b}\right)\right]},
$$

and corresponds to an endemic equilibrium.

The disease is said to be endemic when the force of infection is positive i.e., $\lambda_{b}^{* *}>0$. It is apparent from equation (19) that $\lambda_{b}^{* *}>0$ if and only if $\mathfrak{R}_{0}>1$. The explicit components of the endemic equilibrium, $\mathcal{E}_{1}$, can be determined by substituting (19) into the expressions in (17). We note that, if $\Re_{0}<1$ in (19), then the force of infection, $\lambda_{b}^{* *}$, at equilibrium is negative i.e., $\lambda_{b}^{* *}<0$, which is biologically meaningless and therefore, in this case, the WNV model (4) has no positive endemic equilibrium. The following theorem summarises the existence result.

Theorem 3 The endemic equilibrium, $\mathcal{E}_{1}$, of the WNV model (4) exists and is unique whenever $\mathfrak{R}_{0}>1$, and no endemic equilibrium otherwise.

\section{Stochastic Model}

Stochastic effects are fundamental to all biological systems (Ditlevsen and Adeline 2013). Models with demographic stochasticity describe a discrete movement of individuals between epidemiological classes and not average rates (Bartlett 1960; Lloyd et al. 2007; Maliyoni et al. 2017). The numbers in a stochastic model are integers and not continuously changing values (Lloyd et al. 2007; Maliyoni et al. 2017). In stochastic models, it is possible for the last infective to die or recover before an outbreak occurs and the disease can only reemerge if it is reintroduced by an infective from outside the population (Lahodny and Allen 2013; Lloyd et al. 2007; Maliyoni et al. 2017). A Markov chain model with a discrete number of individuals is, in this case, considered to be more realistic than a deterministic model (Lahodny and Allen 2013). Demographic stochasticity inherent in stochastic models may result in disease dynamics that are different from their analogous deterministic models (Lahodny and Allen 2013; Maliyoni et al. 2017; McCormack and Allen 2005). 


\subsection{CTMC Model Development}

We develop a continuous-time Markov chain (CTMC) model based on the assumptions of the deterministic model (4). For simplicity, we use the same notation for the random variables and parameters as in the deterministic model. Let time be continuous, $t \in[0, \infty)$ and let $S_{b}(t), I_{b}(t), R_{b}(t), S_{m}(t)$, $E_{m}(t)$ and $I_{m}(t)$ denote discrete-valued random variables for the number of susceptible birds, infectious birds, recovered birds, susceptible mosquitoes, exposed mosquitoes, and infectious mosquitoes at time $t$, respectively. The total bird and mosquito population satisfy $N_{b}(t)=S_{b}(t)+I_{b}(t)+R_{b}(t)$ and $N_{m}(t)=S_{m}(t)+E_{m}(t)+I_{m}(t)$, respectively. Let

$$
\{\vec{Y}(t): t \in[0, \infty)\},
$$

where $\vec{Y}(t)=\left[S_{b}(t), I_{b}(t), R_{b}(t), S_{m}(t), E_{m}(t), I_{m}(t)\right]^{T}$, be the associated random vector for all the discrete-valued random variables. The state transition rates for the CTMC are defined in Table 3 .

Table 3 State transitions and rates for the CTMC West Nile virus model

\begin{tabular}{llcc}
\hline Event & State transition $[t \rightarrow(t+\Delta t)]$ & Transition, $\Delta \vec{Y}(t)$ & Transition Rate, $p$ \\
\hline Recruitment of $S_{b}$ & $\left(S_{b}, \ldots\right) \rightarrow\left(S_{b}+1, \ldots\right)$ & $(1,0,0,0,0,0)^{T}$ & $\Lambda_{b}$ \\
Infection of $S_{b}$ & $\left(S_{b}, I_{b}, \ldots\right) \rightarrow\left(S_{b}-1, I_{b}+1, \ldots\right)$ & $(-1,1,0,0,0,0)^{T}$ & $\left(\hat{\beta}_{b} S_{b} I_{m}\right) / N_{b}$ \\
Death of $S_{b}$ & $\left(S_{b}, \ldots\right) \rightarrow\left(S_{b}-1, \ldots\right)$ & $(-1,0,0,0,0,0)^{T}$ & $\mu_{b} S_{b}$ \\
Recovery of $I_{b}$ & $\left(\ldots, I_{b}, R_{b}, \ldots\right) \rightarrow\left(\ldots, I_{b}-1, R_{b}+1, \ldots\right)$ & $(0,-1,1,0,0,0)^{T}$ & $\alpha I_{b}$ \\
Death of $I_{b}$ & $\left(\ldots, I_{b}, \ldots\right) \rightarrow\left(\ldots, I_{b}-1, \ldots\right)$ & $(0,-1,0,0,0,0)^{T}$ & $\left(\delta+\mu_{b}\right) I_{b}$ \\
Death of $R_{b}$ & $\left(\ldots, R_{b}, \ldots\right) \rightarrow\left(\ldots, R_{b}-1, \ldots\right)$ & $(0,0,-1,0,0,0)^{T}$ & $\mu_{b} R_{b}$ \\
Recruitment of $S_{m}$ & $\left(\ldots, S_{m}, \ldots\right) \rightarrow\left(\ldots, S_{m}+1, \ldots\right)$ & $(0,0,0,1,0,0)^{T}$ & $\Lambda_{m}$ \\
Infection of $S_{m}$ & $\left(\ldots, S_{m}, E_{m}, \ldots\right) \rightarrow\left(\ldots, S_{m}-1, E_{m}+1, \ldots\right)$ & $(0,0,0,-1,1,0)^{T}$ & $\left(\hat{\beta}_{m} S_{m} I_{b}\right) / N_{b}$ \\
Death of $S_{m}$ & $\left(\ldots, S_{m}, \ldots\right) \rightarrow\left(\ldots, S_{m}-1, \ldots\right)$ & $(0,0,0,-1,0,0)^{T}$ & $\mu_{m} S_{m}$ \\
Progression of $E_{m}$ & $\left(\ldots, E_{m}, I_{m}\right) \rightarrow\left(\ldots, E_{m}-1, I_{m}+1\right)$ & $(0,0,0,0,-1,1)^{T}$ & $\gamma E_{m}$ \\
Death of $E_{m}$ & $\left(\ldots, E_{m}, \ldots\right) \rightarrow\left(\ldots, E_{m}-1, \ldots\right)$ & $(0,0,0,0,-1,0)^{T}$ & $\mu_{m} E_{m}$ \\
Death of $I_{m}$ & $\left(\ldots, I_{m}\right) \rightarrow\left(\ldots, I_{m}-1\right)$ & $(0,0,0,0,0,-1)^{T}$ & $\mu_{m} I_{m}$ \\
\hline
\end{tabular}

Note: The infinitesimal transition probability for the stochastic process from the state at time $t$ to a new state at time $(t+\Delta t)$, i.e., $\Delta \vec{Y}(t)=\vec{Y}(t+\Delta t)-\vec{Y}(t)$, is given by the expression $p \Delta t+o(\Delta t)$. In the 3rd column, $+1(-1)$ and 0 represent, respectively, an increase (a decrease) by 1 and no change in state for the variable from time $t$ to $(t+\Delta t)$.

We assume that the CTMC model is time-homogeneous and satisfies the Markov property which states that the future state of the process at time $(t+\Delta t)$ depends on the current state at time $t$ only (Allen 2010; Maliyoni et al. 2017; Sani et al. 2006). Owing to the Markov assumption, the time to the next event is exponentially distributed with parameter (Allen 2010; Lahodny and Allen 2013; Lahodny et al. 2015; Maliyoni et al. 2017)

$$
\psi(\vec{Y})=\Lambda_{b}+\mu_{b} N_{b}+(\alpha+\delta) I_{b}+\Lambda_{m}+\mu_{m} N_{m}+\gamma E_{m}+\frac{\hat{\beta}_{b} S_{b} I_{m}+\hat{\beta}_{m} S_{m} I_{b}}{N_{b}} .
$$


To ensure that the transition probabilities of the process are within the interval [0,1], we assume that $\Delta t$ is sufficiently small (Allen 2008; Allen 2010; Allen and Burgin 2000). Besides, we assume that at most one event takes place during the time interval $\Delta t$ (Maliyoni et al. 2017, Maliyoni et al. 2019). The infinitesimal transition probabilities for the events in the CTMC model from the state at time $t$ to a new state at time $(t+\Delta t)$ are defined in Table 3.

Persistence of a disease into a susceptible population is not guaranteed by having $\mathfrak{R}_{0}>1$ because stochastic extinction (also known as stochastic fadeout) can happen as soon as an infective is introduced, when there are few infectives at the beginning of an epidemic process (Lahodny et al. 2015; Lloyd et al. 2007; Maliyoni et al. 2017). In this case, a deterministic model predicts a major outbreak while its corresponding stochastic model predicts a minor outbreak (Lloyd et al. 2007). During the early period when an infective has been introduced, only a few susceptibles get infected as such the probability of disease extinction or outbreak can be approximated from the resulting linear model which assumes that the whole population is susceptible (Allen and Lahodny 2012; Bartlett 1964; Lahodny et al. 2015; Lloyd et al. 2007; Maliyoni et al. 2017; Maliyoni et al. 2019). The theory of multitype branching process is, thus, used to approximate the probability of disease extinction or outbreak.

\subsection{Branching Process Approximation}

The theory of multitype (Galton-Watson) branching process is often used to approximate the dynamics of the nonlinear CTMC near the DFE (Allen and Lahodny 2012; Harris 1963; Lahodny and Allen 2013; Lahodny et al. 2015; Maliyoni et al. 2017). For a few initial infectives, the branching process either grows exponentially or hits zero (Allen 2017). The branching process is applied only to the infectious groups and the initial population(s) is(are) assumed to be at the DFE (Allen and Lahodny 2012; Lahodny and Allen 2013; Lahodny et al. 2015; Maliyoni et al. 2017, Maliyoni et al. 2019). Since the multitype branching process is linear near the DFE, homogeneous in time, and births (i.e., new infections) and deaths are independent, offspring probability generating functions (pgfs) can be defined for the birth and death of the infectious individuals. These offspring pgfs are then used to calculate the probability of disease extinction or a major outbreak (Allen and Lahodny 2012; Lahodny and Allen 2013; Lahodny et al. 2015; Maliyoni et al. 2017, Maliyoni et al. 2019).

Assume that infectious individuals of type $i, I_{i}$, give birth to infectious individuals of type $j, I_{j}$, and that the number of offspring generated by a type $i$ individual does not depend on the number of offspring generated by either type $i$ or type $j$ individuals $j \neq i$ i.e., they are independent (Allen and Lahodny 2012; Lahodny and Allen 2013; Maliyoni et al. 2017). Furthermore, we assume that the initial population in each group is sufficiently large, $S_{b}(0) \approx$ $N_{b}(0), S_{m}(0) \approx N_{m}(0)$ (Lahodny and Allen 2013); and that type $i$ individuals have the same offspring pgf which is independent and identically distributed (iid) (Allen and Lahodny 2012). We define $\left\{Y_{j i}\right\}_{j=1}^{n}$ as the offspring random 
variables for type $i, i=1,2, \ldots, n$ so that $Y_{j i}$ is the number of offspring of type $j$ generated by infectious individuals of type $i$. Let the probability that an individual of type $i$ gives birth to $x_{j}$ individuals of type $j$ be

$$
\mathrm{P}_{i}\left(x_{1}, \ldots, x_{n}\right)=\operatorname{Prob}\left\{Y_{1 i}=x_{1}, \ldots, Y_{n i}=x_{n}\right\},
$$

and, therefore, the offspring pgf for individuals of type $i$ given that $I_{i}(0)=1$ and $I_{j}(0)=0, j \neq i, f_{i}:[0,1]^{n} \rightarrow[0,1]$, is given by (Allen and Lahodny 2012; Allen and van den Driessche 2013; Lahodny and Allen 2013; Maliyoni et al. 2017)

$$
f_{i}\left(u_{1}, \ldots, u_{n}\right)=\sum_{x_{n}=0}^{\infty} \ldots \sum_{x_{1}=0}^{\infty} \mathrm{P}_{i}\left(x_{1}, \ldots, x_{n}\right) u_{1}^{x_{1}} \cdots u_{n}^{x_{n}} .
$$

From equation (22), we define an $n \times n$ nonnegative and irreducible expectation matrix $\mathbb{M}=\left[m_{j i}\right]$, where the element $m_{j i}$ is the expected number of infectious offspring of type $j$ generated by one infectious individual of type $i$ (Allen and Lahodny 2012; Lahodny and Allen 2013; Maliyoni et al. 2017). The elements $m_{j i}$ are determined by (Allen and Lahodny 2012 ;Allen and van den Driessche 2013)

$$
m_{j i}=\left.\frac{\partial f_{i}}{\partial x_{j}}\right|_{\boldsymbol{u}=1}<\infty .
$$

Whether the probability of disease extinction is one or less than one, is determined by the size of the spectral radius of matrix $\mathbb{M}, \rho(\mathbb{M})$ (Allen and Lahodny 2012; Athreya and Ney 1972; Harris 1963; Maliyoni et al. 2017). In particular, if $\rho(\mathbb{M})<1$ or $\rho(\mathbb{M})=1$ (subcritical or critical process, respectively), then the probability of ultimate extinction is one, i.e.,

$$
\lim _{t \rightarrow \infty} \operatorname{Prob}\{\vec{I}(t)=\mathbf{0}\}=1 .
$$

However, if the process is supercritical, that is, if $\rho(\mathbb{M})>1$, then there exists a unique fixed point, $q_{j} \in(0,1)^{n}$, for the offspring pgfs, $f_{i}\left(q_{1}, q_{2}, \ldots, q_{n}\right)=$ $q_{i}$, so that if $i_{j}=I_{j}(0)$, then the probability of ultimate extinction is less than one (Allen 2015; Allen and Lahodny 2012; Allen and van den Driessche 2013;Athreya and Ney 1972; Harris 1963; Lahodny and Allen 2013)

$$
\mathbb{P}_{0}=\lim _{t \rightarrow \infty} \operatorname{Prob}\{\vec{I}(t)=\mathbf{0}\}=q_{1}^{i_{1}} q_{2}^{i_{2}} \ldots q_{n}^{i_{n}}<1 .
$$

Thus, the probability of a major outbreak for the supercritical process is approximately (Allen 2015; Allen 2010; Allen and Lahodny 2012; Allen and van den Driessche 2013; Lahodny and Allen 2013; Lahodny et al. 2015; Maliyoni et al. 2017)

$$
1-q_{1}^{i_{1}} q_{2}^{i_{2}} \ldots q_{n}^{i_{n}} .
$$

The role of the threshold $\rho(\mathbb{M})$ in stochastic models is similar to that of $\Re_{0}$ in deterministic models. Allen and van den Driessche (2013) proved that the 
deterministic and stochastic thresholds for disease extinction, $\mathfrak{R}_{0}$ and $\rho(\mathbb{M})$, respectively, satisfy the following relationship:

$$
\mathfrak{R}_{0}<1,=1,>1 \quad \text { if and only if } \rho(\mathbb{M})<1,=1,>1 .
$$

\subsection{Application of the Branching Process}

In this section, the branching process is applied to the CTMC model and expressions for stochastic threshold for disease extinction and the probability of disease extinction are derived.

\subsubsection{Stochastic Threshold for Disease Extinction}

The multitype branching process is applied to all infected and infectious classes in both populations in the CTMC model i.e., infectious birds, exposed and infectious mosquitoes. Infectious birds (mosquitoes) can generate secondary infectious mosquitoes (birds) while exposed mosquitoes can progress to the infectious class at the beginning of the epidemic process when there are only a few infectives. We define offspring pgfs for these three classes using the formula given in equation (22). We assume that the initial bird and mosquito populations are sufficiently large and are at the disease-free equilibrium i.e., $S_{b}(0)=\frac{\Lambda_{b}}{\mu_{b}}=S_{b}^{*}$ and $S_{m}(0)=\frac{\Lambda_{m}}{\mu_{m}}=S_{m}^{*}$, respectively.

The offspring pgf for $I_{b}$ given that $I_{b}(0)=1, E_{m}(0)=0$ and $I_{m}(0)=0$ is

$$
f_{1}\left(u_{1}, u_{2}, u_{3}\right)=\frac{\bar{\beta}_{m} u_{1} u_{2}+\alpha+\delta+\mu_{b}}{\bar{\beta}_{m}+\alpha+\delta+\mu_{b}} .
$$

The interpretation of the terms in pgf (27) is as follows: an infectious bird infects a susceptible mosquito but the infectious bird does not die which results in two infected individuals i.e., one infectious bird and one exposed mosquito with probability $\bar{\beta}_{m} /\left(\bar{\beta}_{m}+\alpha+\delta+\mu_{b}\right)$. The term $\left(\alpha+\delta+\mu_{b}\right) /\left(\bar{\beta}_{m}+\alpha+\delta+\mu_{b}\right)$ is the probability that an infectious bird may die or recover before transmitting the infection to a susceptible mosquito which results in zero infectious birds and mosquitoes.

Likewise, the offspring pgf for $E_{m}$ given that $I_{b}(0)=0, E_{m}(0)=1$ and $I_{m}(0)=0$ is

$$
f_{2}\left(u_{1}, u_{2}, u_{3}\right)=\frac{\gamma u_{3}+\mu_{m}}{\gamma+\mu_{m}} .
$$

In the pgf (28), the term $\gamma /\left(\gamma+\mu_{m}\right)$ is the probability that an exposed mosquito becomes infectious resulting in zero exposed mosquitoes and one infectious mosquito. $\mu_{m} /\left(\gamma+\mu_{m}\right)$ is the probability that the exposed mosquito may die before becoming infectious which results in zero exposed and infectious mosquitoes.

If $I_{b}(0)=0, E_{m}(0)=0$ and $I_{m}(0)=1$, then the offspring pgf for $I_{m}$ is given by 


$$
f_{3}\left(u_{1}, u_{2}, u_{3}\right)=\frac{\hat{\beta}_{b} u_{1} u_{3}+\mu_{m}}{\hat{\beta}_{b}+\mu_{m}} .
$$

In the pgf $(29), \hat{\beta}_{b} /\left(\hat{\beta}_{b}+\mu_{m}\right)$ represents the probability that an infectious mosquito infects a susceptible bird but the infectious mosquito survives which results in two infectious individuals i.e., one infectious mosquito and one infectious bird. The probability that the infectious mosquito may also die before transmitting the infection to a susceptible bird resulting in zero infectious mosquitoes and birds, is $\mu_{m} /\left(\hat{\beta}_{b}+\mu_{m}\right)$.

The expectation matrix, $\mathbb{M}$ of the branching process is a $3 \times 3$ matrix which is determined by using equation (23), offspring pgfs (27)-(29) and evaluated at $\boldsymbol{u}=\left(u_{1}, u_{2}, u_{3}\right)=1$. Thus,

$$
\mathbb{M}=\left.\left[\begin{array}{lll}
\frac{\partial f_{1}}{\partial u_{1}} & \frac{\partial f_{2}}{\partial u_{1}} & \frac{\partial f_{3}}{\partial u_{1}} \\
\frac{\partial f_{1}}{\partial u_{2}} & \frac{\partial f_{2}}{\partial u_{2}} & \frac{\partial f_{3}}{\partial u_{3}} \\
\frac{\partial f_{1}}{\partial u_{3}} & \frac{\partial f_{2}}{\partial u_{3}} & \frac{\partial f_{3}}{\partial u_{3}}
\end{array}\right]\right|_{\boldsymbol{u}=\mathbf{1}}=\left[\begin{array}{ccc}
\frac{\bar{\beta}_{m}}{\bar{\beta}_{m}+\alpha+\delta+\mu_{b}} & 0 & \frac{\hat{\beta}_{b}}{\hat{\beta}_{b}+\mu_{m}} \\
\overline{\bar{\beta}_{m}+\alpha+\delta+\mu_{b}} & 0 & 0 \\
0 & \frac{\gamma}{\gamma+\mu_{m}} & \frac{\hat{\beta}_{b}}{\hat{\beta}_{b}+\mu_{m}}
\end{array}\right]
$$

The elements $m_{11}, m_{21}$ and $m_{31}$ represent the expected number of infectious birds, exposed and infectious mosquitoes, respectively, generated by one infectious bird. Likewise, the elements $m_{12}, m_{22}$ and $m_{32}$ represent the expected number of infectious birds, exposed and infectious mosquitoes, respectively, generated by one exposed mosquito. The elements $m_{13}, m_{23}$ and $m_{33}$ represent the expected number of infectious birds, exposed and infectious mosquitoes, respectively, generated by one infectious mosquito.

The threshold for disease extinction or persistence for the CTMC model is the spectral radius of matrix $\mathbb{M}, \rho(\mathbb{M})$, which is given by

$$
\rho(\mathbb{M})=\frac{\gamma \bar{\beta}_{m}\left(\hat{\beta}_{b}+\mu_{m}\left(\gamma+\mu_{m}\right)\right)+\hat{\beta}_{b}\left(\gamma \bar{\beta}_{m}+\alpha+\delta+\mu_{b}\right)}{\left(\gamma \bar{\beta}_{m}+\alpha+\delta+\mu_{b}\right)\left(\hat{\beta}_{b}+\mu_{m}\left(\gamma+\mu_{m}\right)\right)} .
$$

It can easily be verified that equation $(26)$ holds for $\rho(\mathbb{M})$.

\subsubsection{Expression for Probability of Disease Extinction}

For the supercritical branching process i.e., $\rho(\mathbb{M})>1$ and $\mathfrak{R}_{0}>1$, there exists a fixed point, $\left(q_{1}, q_{2}, q_{3}\right) \in(0,1)^{3}$ of the offspring pgfs $(27)-(29)$ which is used to write the explicit expression for the probability of disease extinction (Allen and Lahodny 2012; Allen and van den Driessche 2013; Maliyoni et al. 2017). To obtain the fixed point, we set $f_{i}\left(q_{1}, q_{2}, q_{3}\right)=q_{i}$, for $i=1,2,3$. The solutions of this system are $(1,1,1)$ and $\left(q_{1}, q_{2}, q_{3}\right)$ (Allen 2017; Allen 2010; Allen and Lahodny 2012; Maliyoni et al. 2019). Thus, we consider the following system of equations: 


$$
\begin{array}{ll}
\frac{\bar{\beta}_{m} q_{1} q_{2}+\alpha+\delta+\mu_{b}}{\bar{\beta}_{m}+\alpha+\delta+\mu_{b}} & =q_{1}, \\
\frac{\gamma q_{3}+\mu_{m}}{\gamma+\mu_{m}} & =q_{2}, \\
\frac{\hat{\beta}_{b} q_{1} q_{3}+\mu_{m}}{\hat{\beta}_{b}+\mu_{m}} & =q_{3} .
\end{array}
$$

Solving system (32) for $q_{1}, q_{2}$ and $q_{3}$, respectively, gives

$$
\begin{aligned}
q_{1} & =\frac{\gamma \bar{\beta}_{m}}{\gamma \bar{\beta}_{m}+\left(\gamma+\mu_{m}\right)\left(\alpha+\delta+\mu_{b}\right)}\left(\frac{1}{\mathfrak{R}_{0}^{2}}\right)+\frac{\left(\gamma+\mu_{m}\right)\left(\alpha+\delta+\mu_{b}\right)}{\gamma \bar{\beta}_{m}+\left(\gamma+\mu_{m}\right)\left(\alpha+\delta+\mu_{b}\right)}, \\
q_{2} & =\frac{\gamma}{\gamma+\mu_{m}}\left(\frac{\hat{\beta}_{b}}{\hat{\beta}_{b}+\mu_{m}}\left(\frac{1}{\mathfrak{R}_{0}^{2}}\right)+\frac{\mu_{m}}{\hat{\beta}_{b}+\mu_{m}}\right)+\frac{\mu_{m}}{\gamma+\mu_{m}}, \\
q_{3} & =\frac{\hat{\beta}_{b}}{\hat{\beta}_{b}+\mu_{m}}\left(\frac{1}{\mathfrak{R}_{0}^{2}}\right)+\frac{\mu_{m}}{\hat{\beta}_{b}+\mu_{m}} .
\end{aligned}
$$

The terms in the expression of $q_{1}$ have the following biological interpretation: an infectious bird will either transmit the infection to a susceptible mosquito with probability $\gamma \bar{\beta}_{m} /\left[\gamma \bar{\beta}_{m}+\left(\gamma+\mu_{m}\right)\left(\alpha+\delta+\mu_{b}\right)\right]$ or may die or recover before transmission with probability $\left(\gamma+\mu_{m}\right)\left(\alpha+\delta+\mu_{b}\right) /\left[\gamma \bar{\beta}_{m}+\right.$ $\left.\left(\gamma+\mu_{m}\right)\left(\alpha+\delta+\mu_{b}\right)\right]$. Likewise, the terms in $q_{3}$ can be interpreted as follows: an infectious mosquito will either transmit the infection to a susceptible bird with probability $\hat{\beta}_{b} /\left(\hat{\beta}_{b}+\mu_{m}\right)$ or may die before transmitting the disease with probability $\mu_{m} /\left(\hat{\beta}_{b}+\mu_{m}\right)$. If disease transmission from an infectious bird (mosquito) to a susceptible mosquito (bird) is successful, then the probability of transmission is $1-\left(1 / \mathfrak{R}_{0}^{2}\right)$. The value of $q_{2}$ can be interpreted as follows: an exposed mosquito either survives to become infectious with probability $\gamma /\left(\gamma+\mu_{m}\right)$ or dies with probability $\mu_{m} /\left(\gamma+\mu_{m}\right)$.

Employing the expressions for $q_{1}, q_{2}$ and $q_{3}$, the probability of West Nile virus extinction, given that the initial number of infectious birds, exposed and infectious mosquitoes are $I_{b}(0)=i_{b}, E_{m}(0)=e_{m}$ and $I_{m}(0)=i_{m}$, respectively, is approximately (Allen and Lahodny 2012; Lahodny and Allen 2013; Maliyoni et al. 2017; 2019)

$$
\begin{aligned}
\mathbb{P}_{0}= & q_{1}^{i_{b}} \times q_{2}^{e_{m}} \times q_{3}^{i_{m}}, \\
= & {\left[\frac{\gamma \bar{\beta}_{m}}{\gamma \bar{\beta}_{m}+\left(\gamma+\mu_{m}\right)\left(\alpha+\delta+\mu_{b}\right)}\left(\frac{1}{\mathfrak{R}_{0}^{2}}\right)+\frac{\left(\gamma+\mu_{m}\right)\left(\alpha+\delta+\mu_{b}\right)}{\gamma \bar{\beta}_{m}+\left(\gamma+\mu_{m}\right)\left(\alpha+\delta+\mu_{b}\right)}\right]^{i_{b}} } \\
& \times\left[\frac{\gamma}{\gamma+\mu_{m}}\left(\frac{\hat{\beta}_{b}}{\hat{\beta}_{b}+\mu_{m}}\left(\frac{1}{\mathfrak{R}_{0}^{2}}\right)+\frac{\mu_{m}}{\hat{\beta}_{b}+\mu_{m}}\right)+\frac{\mu_{m}}{\gamma+\mu_{m}}\right]^{e_{m}} \\
& \times\left[\frac{\hat{\beta}_{b}}{\hat{\beta}_{b}+\mu_{m}}\left(\frac{1}{\mathfrak{R}_{0}^{2}}\right)+\frac{\mu_{m}}{\hat{\beta}_{b}+\mu_{m}}\right]^{i_{m}}
\end{aligned}
$$


and the probability of an outbreak (or persistence) is

$$
1-\mathbb{P}_{0} \text {. }
$$

\section{Numerical Simulations}

The dynamics of the deterministic and CTMC epidemic models for West Nile virus are illustrated numerically. An approximation of the probability of disease extinction or a major outbreak and the finite-time to disease extinction is carried out. All results in this section are based on the parameter values given in Table 4 . For these parameter values, the basic reproduction number $\mathfrak{R}_{0}=1.7516$ and the extinction threshold for the CTMC model is $\rho(\mathbb{M})=1.0896$.

Table 4 Model parameter values (for both birds and mosquitoes) used in numerical simulations. All rates are per capita per day $\left(\right.$ day $\left.^{-1}\right)$

\begin{tabular}{cccl}
\hline Parameter & Value & Range & Reference(s) \\
\hline$\Lambda_{b}$ & 1.00 & - & Assumed \\
$\Lambda_{m}$ & 50.0 & - & Assumed \\
$\beta_{b}$ & 0.35 & $0.27-1.00$ & Wonham and Lewis (2008) \\
$\beta_{m}$ & 0.25 & $0.23-1.00$ & Wonham and Lewis (2008) \\
$\sigma_{b}$ & 0.35 & $0.34-0.53$ & Wonham and Lewis (2008) \\
$\mu_{b}$ & 0.01 & $0.00-0.01$ & Botkin and Miller (1974); Simpson et al. (2008) \\
$\mu_{m}$ & 0.05 & $0.02-0.07$ & Wonham and Lewis (2008) \\
$\alpha$ & 0.20 & $0.00-0.20$ & Bergsman et al. (2016); Komar (2003) \\
$\gamma$ & 0.08 & $0.07-0.14$ & Bergsman et al. (2016); Sardelis et al. (2001) \\
$\delta$ & 0.22 & $0.17-0.25$ & Wonham and Lewis (2008) \\
\hline
\end{tabular}

\subsection{Probability of Disease Extinction or a Major Outbreak}

The probability of disease extinction, $\mathbb{P}_{0}$, is calculated from the fixed point of the multitype branching process, particularly equation (34). $\mathbb{P}_{0}$ is compared to the approximated probability of disease extinction, $\mathbb{P}_{A}$, obtained from a proportion of 10,000 sample paths of the WNV CTMC model for which the total number of infected individuals, that is, $\left(I_{b}(t)+E_{m}(t)+I_{m}(t)\right)$ equals zero before reaching a minimum outbreak size. Table 5 shows that $\mathbb{P}_{0}$ and $\mathbb{P}_{A}$ are in good agreement. The fixed point in $(0,1)^{3}$ is given by $\left(q_{1}, q_{2}, q_{3}\right)=$ $(0.6252,0.7054,0.5213)$.

The results in Table 5 show a high probability of disease extinction if there is an exposed mosquito only at the beginning of an epidemic; thereby reducing the likelihood of a major disease outbreak. This is attributed to the fact that exposed mosquitoes do not transmit the disease unless they survive and become infectious. For infectious individuals, $\mathbb{P}_{0}$ is smaller if the disease is introduced by an infectious mosquito and not by an infectious bird. Thus, a disease 
Table 5 Probability of disease extinction $\mathbb{P}_{0}$ for West Nile virus calculated from the fixed point of the branching process and numerical approximation $\mathbb{P}_{A}$ based on 10,000 sample paths of the CTMC model. Parameter values are as in Table 4 with initial conditions $S_{b}(0)=$ $S_{b}^{*}=100, R_{b}(0)=0, S_{m}(0)=S_{m}^{*}=1000, I_{b}(0)=i_{b}, E_{m}(0)=e_{m}$ and $I_{m}(0)=i_{m}$. The basic reproduction number $\mathfrak{R}_{0}=1.7516$ and $\rho(\mathbb{M})=1.0896$

\begin{tabular}{ccccc}
\hline$i_{b}$ & $e_{m}$ & $i_{m}$ & $\mathbb{P}_{0}$ & $\mathbb{P}_{A}$ \\
\hline 1 & 0 & 0 & 0.6252 & 0.6229 \\
0 & 1 & 0 & 0.7054 & 0.7061 \\
0 & 0 & 1 & 0.5213 & 0.5281 \\
2 & 0 & 0 & 0.3909 & 0.3863 \\
0 & 2 & 0 & 0.4976 & 0.4971 \\
0 & 0 & 2 & 0.2718 & 0.2713 \\
1 & 1 & 1 & 0.2299 & 0.2289 \\
2 & 2 & 2 & 0.0529 & 0.0531 \\
\hline
\end{tabular}

outbreak is likely if infectious mosquitoes introduce the disease in an entirely susceptible bird population. Also, the probability of disease outbreak is very high if all 3 infected groups are present at the onset of the epidemic process. If a disease outbreak occurs (i.e., the basic reproduction number is greater than unity, $\mathfrak{R}_{0}>1$ ), then it is possible for the disease to fade-out or persist in the CTMC model during the early days of the epidemic. Unlike in deterministic models, disease extinction in stochastic models can occur even if $\mathfrak{R}_{0}>1$ due to demographic stochasticity that is inherent in the system; which is one of the major asymptotic differences between deterministic and stochastic models ((Allen 2017; Allen 2008; Maliyoni et al. 2017; McCormack and Allen 2005). Based on these results, it is clear that WNV can be contained if mosquito population is reduced by implementing various intervention strategies such as spraying, larviciding (killing mosquito larvae), adulticiding (killing adult mosquitoes), as suggested by Bergsman et al. (2016) and Chen et al. (2016). Figures 2 and 3 illustrate disease persistence and extinction, respectively, in the CTMC model when $\mathfrak{R}_{0}>1$. The deterministic and stochastic solutions are plotted on the same graph for easy comparison.

\subsection{Finite-Time Extinction}

Stochastic models are unique in that the finite-time to disease extinction (also known as expected epidemic duration) $T$ can be estimated; a property that deterministic models do not exhibit (Allen 2008; Lahodny and Allen 2013; Maliyoni et al. 2019). The finite-time extinction corresponds to the time $T$ until the number of infectious individuals asymptotically approaches zero (Allen 2008; Lahodny and Allen 2013; Maliyoni et al. 2019; Mollison 1991). The length of $T$ depends on the initial number of infected individuals, the population size, and the value of the basic reproduction number (Allen 2008). Table 6 indicate the estimated finite-time extinction for West Nile virus while Figure 4 shows the approximate distribution of $T$. Values of $T$ in Table 6 are estimated using 
(a)

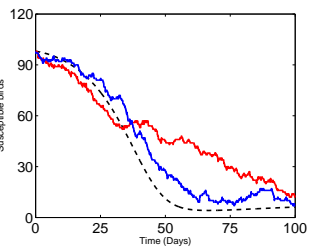

(d)

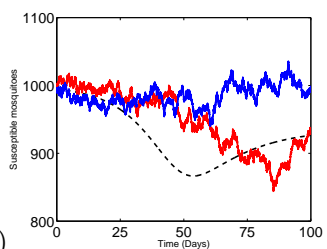

(b)

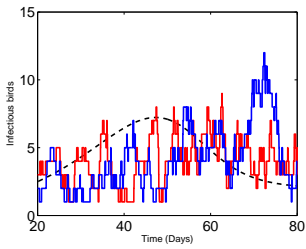

(e)

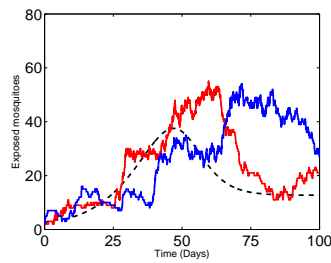

(c)

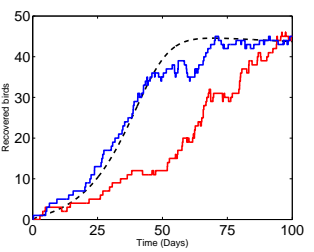

(f)

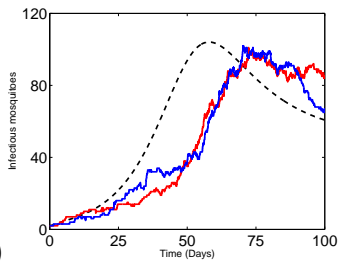

Fig. 2 Comparison of two sample paths of the CTMC model and the deterministic solution (dashed curve) illustaring a major outbreak and disease persistence. Parameter values are as in Table 4 with initial conditions $S_{b}(0)=100, I_{b}(0)=2, R_{b}(0)=0, S_{m}(0)=1000$, $E_{m}(0)=2$ and $I_{m}(0)=2$. The probability of an outbreak is $1-\mathbb{P}_{0}=0.9471$ (see Table 5 ), $\mathfrak{R}_{0}=1.7516$ and $\rho(\mathbb{M})=1.0896$

(a)

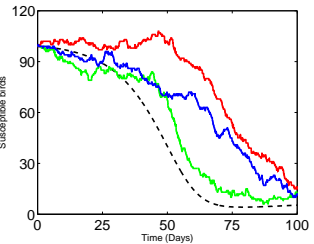

(d)

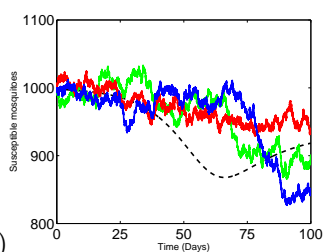

(b)

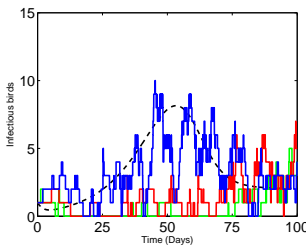

(e)

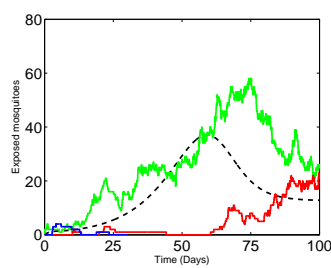

(c)

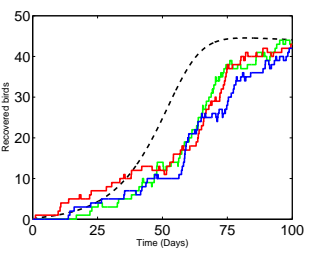

(f)

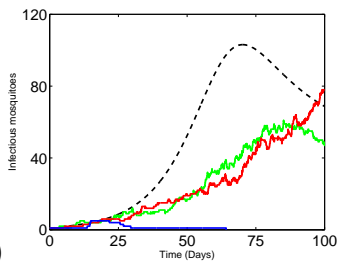

Fig. 3 Comparison of three sample paths of the CTMC model and the deterministic solution (dashed curve) illustaring the possibility of disease extinction (indicated by sample paths of infectious birds, exposed and infectious mosquitoes that hit the time axis in finite time). Parameter values are as in Table 4 with initial conditions $S_{b}(0)=100, I_{b}(0)=1, R_{b}(0)=0$, $S_{m}(0)=1000, E_{m}(0)=0$ and $I_{m}(0)=1$. The probability of disease extinction is 0.3259 , $\mathfrak{R}_{0}=1.7516$ and $\rho(\mathbb{M})=1.0896$

10,000 sample paths and 95th percentile of end times as indicators of epidemic duration.

Table 6 shows that time to disease extinction is shortest if the disease is introduced by exposed mosquitoes since these do not transmit the disease unless they survive the latent period and progress to the infectious stage. This result agrees with the results in Table 5 which suggest that the probability of disease extinction is highest if the disease emerges from exposed mosquitoes. Thus, 
Table 6 Time of disease extinction $T$ for the CTMC model when the infection is introduced by an infectious bird, exposed mosquito, infectious mosquito, and all infected individuals. $T$ is estimated based on 10,000 sample paths and 95th percentile of end times. Parameter values are as in Table 4. The reproduction number is $\mathfrak{R}_{0}=1.7516$ and $\rho(\mathbb{M})=1.0896$

\begin{tabular}{ccc}
\hline Group(s) introducing the disease & $\mathbb{P}_{0}$ & $T$ (days) \\
\hline$I_{b}$ & 0.6252 & 36.2 \\
$E_{m}$ & 0.7054 & 33.7 \\
$I_{m}$ & 0.5213 & 36.5 \\
$I_{b}, E_{m}, I_{m}$ & 0.2299 & 38.1 \\
\hline
\end{tabular}

(a)

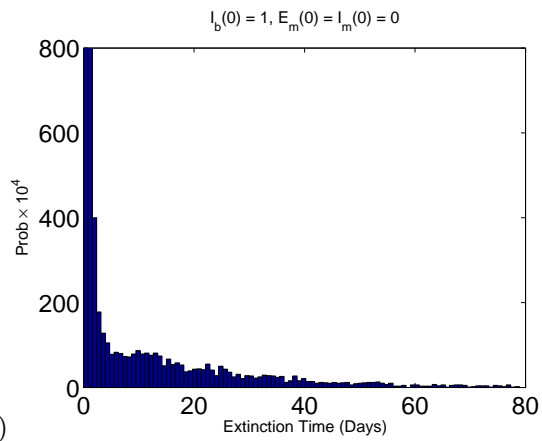

(c)

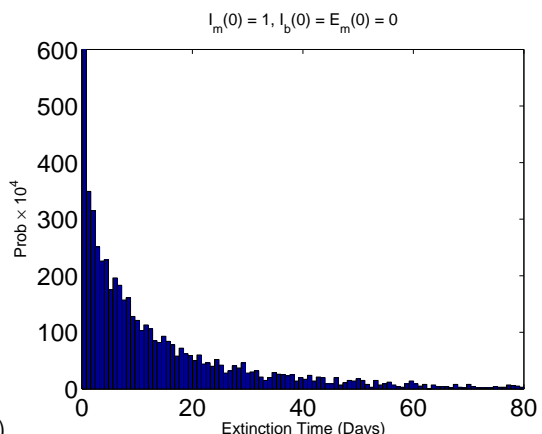

(b)

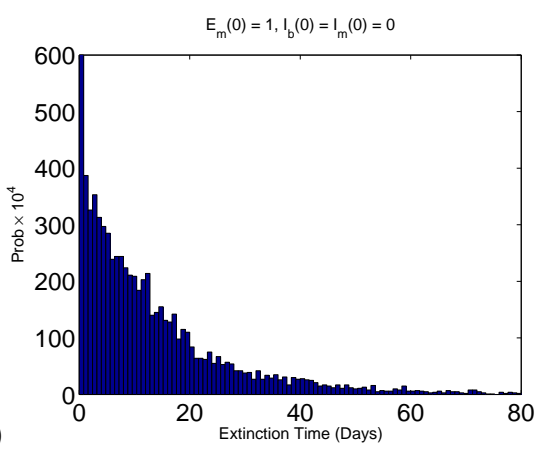

(d)

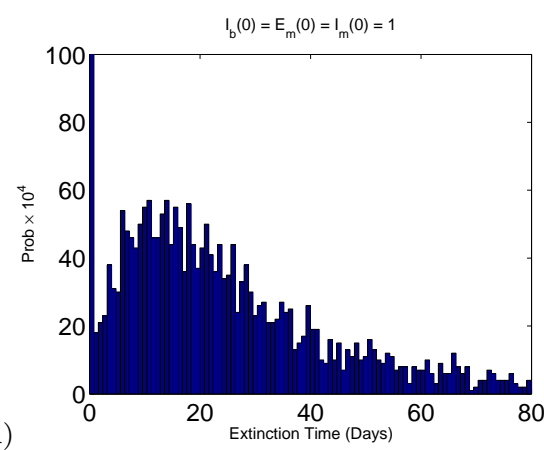

Fig. 4 Approximate probability distribution for the time of disease extinction $T$ for the CTMC model when the disease is introduced by an infectious bird, exposed mosquito, infectious mosquito, and all infected individuals. Parameter values are as in Table 4 and initial conditions are indicated above each graph. Probability of disease extinction $\mathbb{P}_{0}$ and $T$ for each graph are presented in Table 6 . The reproduction number is $\Re_{0}=1.7516$ and stochastic threshold for disease extinction is $\rho(\mathbb{M})=1.0896$. Histograms are plotted based on 10,000 sample paths

the higher the probability of extinction, the shorter the epidemic duration. It can also be observed from Table 6 that the epidemic duration is longest if all infected individuals introduce the disease at the onset of the epidemic process as also predicted by the branching process (see Table 5). Figure 4 (a), (b), (c), and (d) show the approximate probability distribution of epidemic extinction times when the disease is introduced by an infectious bird, exposed mosquito, 
infectious mosquito, and all infected individuals, respectively. The mass of the probability distributions in Figure 4 that make up the early disease extinctions corresponds to the probability of disease extinction as predicted by the branching process (see Table 5).

\section{Summary}

West Nile virus (WNV) is an emerging mosquito-borne virus that is of public health concern throughout the world because it causes significant illness and may become fatal if not treated. Despite various mosquito control programmes and sensitization campaigns, controlling or eliminating mosquitoes, which are vectors for WNV, remains a challenge as outbreaks of WNV continue to be registered. In this paper, a continuous-time Markov chain (CTMC) model was formulated and analysed to ascertain the effect of demographic stochasticity on the transmission dynamics of WNV in birds. The deterministic model is an extension of the model (with larval class) by Wonham and Lewis (2008). For a deterministic model, the extinction threshold provides essential information about disease extinction or persistence. However, its analogous stochastic model does not only provide conditions for disease extinction or persistence but also provides the probability of occurrence of these outcomes by applying the branching process (Allen and van den Driessche 2013; Lahodny and Allen 2013; Maliyoni et al. 2017; Maliyoni et al. 2019). The existence and stability conditions for the deterministic model equilibria are presented. It is shown that the disease-free and the endemic equilibria exist whenever $\mathfrak{R}_{0}<1$ and $\mathfrak{R}_{0}>1$, respectively.

The branching process was applied to the CTMC model to derive the stochastic threshold for disease extinction as well as an explicit expression that was used to compute the probability of disease extinction or outbreak (see equations 31, 34 and 35). Extinction thresholds for the deterministic model $\left(\mathfrak{R}_{0}\right)$ and stochastic model $(\rho(\mathbb{M}))$ are computed and the relationship that exists between them is presented (see equation 26). It was shown that for $\mathfrak{R}_{0}<1$ and $\rho(\mathbb{M})<1$, both models predict disease extinction with certainty. However, the asymptotic behaviour between the two models is significantly different when $\mathfrak{R}_{0}>1$ and $\rho(\mathbb{M})>1$. Thus, the deterministic model predicts disease outbreak (see Figure 2) while its corresponding stochastic model predicts either disease extinction or disease outbreak (see Figure 3). This behaviour is attributed to demographic variability that is inherent in the CTMC model and makes it possible for disease extinction to occur prior to a major outbreak (Lahodny et al. 2015; Kirupaharan and Allen 2004; Maliyoni et al. 2019).

Analytical and numerical results showed a good agreement between the probability of disease extinction $\mathbb{P}_{0}$ calculated from the branching process and the probability estimated from numerical simulations using 10,000 sample paths (see Table 5). Further, it was observed from Table 5 that $\mathbb{P}_{0}$ is high if an exposed mosquito introduces the disease at the beginning of the 
epidemic process which reduces the chances of a disease outbreak. This observation is consistent with the expectation and the fact that exposed mosquitoes do not transmit the disease unless they survive and become infectious. Thus, mosquitoes' survival rate and subsequent progress to the infectious state are very crucial in the WNV disease dynamics. The results also suggest that $\mathbb{P}_{0}$ is smaller if the disease is introduced by an infectious mosquito and not by an infectious bird. Thus, the probability of disease outbreak is high if an infectious mosquito introduces the disease in a wholly susceptible bird population. It was also noted that the probability of disease outbreak is very high if the disease is introduced by all three infected groups at the beginning of the epidemic. The results suggest that interventions to mitigate the impact of WNV should aim at reducing or eliminating the mosquito population size. Thus, there is a need to employ control strategies that will increase the natural death rate of mosquitoes. These control strategies include but not limited to usage of adulticide and larvicide in flooded breeding habitats to prevent and control mosquito emergence (Mpeshe et al. 2011). These results agree with the results of Bergsman et al. (2016) who explored the impact of reducing the mosquito population, for instance, through spraying, and found that decreasing the mosquito-to-bird ratio from 10:1 to 4:1 significantly decelerated and lessened the summer outbreak of WNV in North America. In addition, Chen et al. (2016) argued that increasing the death rate of mosquitoes, which is largely achieved by adulticiding, works immediately to control WNV. Mosquito population control is effective for controlling WNV and preventing major outbreaks when a combination of the larval and adult mosquito control strategies are implemented concurrently (Chen et al. 2016).

Finite-time to disease extinction $T$ was estimated using 10,000 sample paths and the 95th percentile of end times as indicators of epidemic duration in numerical simulations. It was observed that time to disease extinction is the shortest if the disease is introduced by exposed mosquitoes because exposed mosquitoes do not transmit the disease unless they become infectious (see Table 6) which is in agreement with the result that the probability of disease extinction is highest if the disease is introduced by an exposed mosquito (see Table 5). However, it was shown that $T$ is longest if all infected groups introduce the disease at the start of the epidemic as also predicted by the branching process (see Table 5). It is worth mentioning that the model parameters used in this paper were only estimates or assumptions from previous studies on WNV due to lack of true values of the same. To effectively guide public health policy makers and other stakeholders, prediction of disease extinction or outbreak which is crucial for disease prevention and control could greatly improve if accurate parameter values or real data for WNV outbreak are obtained and tested. In addition, the effectiveness of various WNV control strategies particularly those that aim at reducing the mosquito population can be assessed using the probability of disease extinction and real data. However, the results in this paper have provided significant insights into the dynamics of WNV in birds especially that the model has accounted for demographic stochasticity that occurs in the system. Further insights about WNV dynam- 
ics can be achieved by considering environmental stochasticity in the model since mosquitoes are affected by seasonal or environmental variations.

Acknowledgements I am grateful to the University of Malawi for sponsoring my doctoral studies at the University of KwaZulu-Natal (UKZN), South Africa where I was introduced to stochastic modelling. I extend my gratitude to Dr. Faraimunashe Chirove (UKZN), Professor Keshlan S. Govinder (UKZN) and Professor Holly D. Gaff (Old Dominion University, USA) for mentoring me when I was their PhD student.

\section{References}

1. Allen LJS (2017) A primer on stochastic epidemic models: Formulation, numerical simulation, and analysis. Infect Dis Modell 2:128-142

2. Allen LJS (2015) Stochastic population and epidemic models: persistence and extinction. Mathematical Biosciences Institute lecture series, stochastics in biological systems, vol 1.3. Springer International Publishing, Berlin

3. Allen LJS (2010) An introduction to stochastic processes with applications to biology, 2nd edn. Chapman and Hall/CRC Press, Boca Raton

4. Allen LJS (2008) An introduction to stochastic epidemic models. In: Brauer F, van den Driessche P, Wu J (eds) Mathematical epidemiology. Springer, Berlin, pp 77-128

5. Allen LJS, Burgin AM (2000) Comparison of deterministic and stochastic SIS and SIR models in discrete time. Math Biosci 163:1-34

6. Allen LJS, Lahodny GE Jr (2012) Extinction thresholds in deterministic and stochastic epidemic models. J Biol Dyn 6:590-611

7. Allen LJS and van den Driessche P (2013) Relations between deterministic and stochastic thresholds for disease extinction in continuous- and discrete-time infectious disease models. Math Biosci 243:99-108

8. Anderson RM, May RM (1991) Infectious diseases of humans: dynamics and control, 2nd edn. Oxford University Press, London

9. Athreya KB, Ney PE (1972) Branching process. Springer, New York

10. Bartlett MS (1960) Stochastic population models. Methuen, London

11. Bartlett MS (1964) The relevance of stochastic models for large-scale epidemiological phenomena. Appl Stat 13:2-8. doi:10.2307/2985217

12. Bergsman LD, Hyman JM, Manore CA (2016) A mathematical model for the spread of West Nile Virus in migratory and resident birds. Math Biosci Eng. doi: $10.3934 / \mathrm{mbe} .2015009$

13. Botkin DB, Miller RS (1974) Mortality rates and survival of birds. Am Nat 108:181-192

14. Bowman C, Gumel A, van den Driessche P, Wu J, Zhu H (2005) A mathematical model for assessing control strategies against West Nile virus. Bull Math Biol 67:1107-1133

15. Bunning ML, Bowen RA, Cropp B, Sullivan K, Davis B, Komar N, Godsey M, Baker D, Jetter D, Holmes D et al. (2001) Experimental infection of horses with West Nile virus and their potential to infect mosquitoes and serve as amplifying hosts. Ann N Y Acad Sci 951:338-339

16. Center for Disease Control and Prevention, Statistics, surveillance, and control archive. http://www.cdc.gov/ncidod/dvbid/westnile/surv\&control_archive.htm. Accessed: August 04, 2018

17. Center for Disease Control and Prevention, West Nile virus questions and answers. http://www.cdc.gov/ncidod/dvbid/westnile/qa/pesticides.htm. Accessed: August 15,2018

18. Chatterjee S, Pal S, Chattopadhyay J (2008) Role of migratory birds under environmental fluctuation: a mathematical study. J Biol Syst 16:81-106

19. Chen J, Huang J, Beier JC, Cantrell RS, Cosner C, Fuller DO, Zhang G, Ruan S (2016) Modeling and control of local outbreaks of West Nile Virus in the United States. Discrete Continuous Dyn Syst Ser B 21(8):2423-2449 
20. Chevalier V, Annelise T, Benoit D (2014) Predictive modeling of West Nile virus transmission risk in the Mediterranean basin: How far from landing? Int J Environ Res Public Health 11:67-90

21. Cruz-Pacheco G, Esteva L, Montao-Hirose J, Vargas C (2005) Modelling the dynamics of West Nile virus. Bull Math Biol 67:1157-1172

22. Diekmann O, Heesterbeek JA, Metz JA (1990) On the definition and the computation of the basic reproduction ratio $R_{0}$ in models for infectious diseases in heterogeneous populations. J Math Biol 28(4):365-382

23. Ditlevsen S, Adeline S (2013) Introduction to stochastic models in Biology. In: Bachar M, et al. (eds) Stochastic biomathematical models, lecture notes in Mathematics 2058. Springer-Verlag Berlin Heidelberg. DOI 10.1007/978-3-642-32157-3_1

24. Eidson M, Kramer L, Stone W, Hagiwara Y, Schmit K, New York State West Nile virus Avian Surveillance Team. Dead bird surveillance as an early warning system for West Nile virus. Emerg Infect Dis 7:631-635

25. Harris TE (1963) The Theory of Branching Processes. Springer-Perlag, Berlin

26. Hayes EB, Gubler DJ (2006) West Nile virus: Epidemiology and clinical features of an emerging epidemic in the United States. Annu Rev Med 57:181-194

27. Hethcote HW (2000) The mathematics of infectious diseases. SIAM Rev 42:599-653

28. Kirupaharan N, Allen LJS (2004) Coexistence of multiple pathogen strains in stochastic epidemic models with density-dependent mortality. Bull Math Biol 66:841-864

29. Komar N (2003) West nile virus: epidemiology and ecology in North America. Adv Virus Res 61:185-234

30. Lahodny GE Jr, Allen LJS (2013) Probability of a disease outbreak in stochastic multipatch epidemic models. Bull Math Biol. doi:10.1007/s11538-013-9848-z

31. Lahodny GE Jr, Gautam R, Ivanek R (2015) Estimating the probability of an extinction or major outbreak for an environmentally transmitted infectious disease. J Biol Dyn 9:128155

32. Laperriere V, Brugger K, Rubel F (2011) Simulation of the seasonal cycles of bird, equine and human West Nile virus cases. Prev Vet Med 98:99-110

33. Lloyd AL, Zhang J, Root AM (2007) Stochasticity and heterogeneity in host-vector models. J R Soc Interface 4:851-863

34. Mackenzie J, Gubler D, Petersen L (2004) Emerging flaviviruses: The spread and resurgence of Japanese encephalitis, West Nile and dengue viruses. Nat Med 10:S98-S109

35. Maliyoni M, Chirove F, Gaff HD, Govinder KS (2019) A stochastic epidemic model for the dynamics of two pathogens in a single tick population. Theor Popul Biol 127:75-90

36. Maliyoni M, Chirove F, Gaff HD, Govinder KS (2017) A stochastic tick-borne disease model: Exploring the probability of pathogen persistence. Bull Math Biol 79:1999-2021

37. Marfin AA, Gubler DJ (2001) West Nile encephalitis: An emerging disease in the United States. Clin Infect Dis 33:1712-1719

38. McCormack RK, Allen LJS (2005) Disease emergence in deterministic and stochastic models for host and pathogen. Appl Math Comput 168:1281-1305

39. Mollison D (1991) Dependence of epidemic and population velocities on basic parameters. Math Biosci 107:255-287

40. Mpeshe SC, Haario H, Tchuenche JM (2011) A mathematical model of Rift Valley fever with human host. Acta Biotheor 59:231-250

41. Mwamtobe PM, Simelane SM, Abelman S, Tchuenche JM (2017) Mathematical analysis of a lymphatic filariasis model with quarantine and treatment. BMC Public Health 17:265

42. Petersen LR, Roehrig JT (2001) West Nile virus: a reemerging global pathogen. Emerg Infect Dis 7:611-614

43. Qiu Z (2011) Dynamics of an epidemic model with host migration Appl Math Comput 218:4614-4625

44. Rossi S, Ross T, Evans J (2010) West Nile virus. Clin Lab Med 30:47-65

45. Sambri V, Capobianchi M, Charrel R, Fyodorova M, Gaibani P, Gould E, Niedrig M, Papa A, Pierro A, Rossini G, et al. (2013) West Nile virus in Europe: Emergence, epidemiology, diagnosis, treatment, and prevention. Clin Microbiol Infect 19:699-704

46. Sani A, Kroese DP, Pollett PK (2006) Stochastic models for the spread of HIP in a mobile heterosexual population. Math Biosci. doi:10.1016/j.mbs.2006.09.024 
47. Sardelis MR, Turell MJ, Dohm DJ, O'Guinn ML (2001) Vector competence of selected North American culex and coquillettidia mosquitoes for west nile virus. Emerg Infect Dis 7(6):1018-22

48. Simpson JE, Hurtado PJ, Medlock J, Molaei G, Andreadis TG, Galvani AP, DiukWasser MA (2008) Vector host-feeding preferences drive transmission of multi-host pathogens: West nile virus as a model system. Proc R Soc Lond B Biol Sci 279:925-933

49. Steele KE, Linn MJ, Schoepp RJ, Komar N, Geisbert TW, Manduca RM, Calle PR, Raphael BL, Clippinger TL, Larsen T, Smith J, Lanciotti RS, Panella NA, Mc Namara TS (2000). Pathology of fatal West Nile virus infections in native and exotic birds during the 1999 outbreak in New York City. Vet Pathol 37:208-224

50. Thomas DM, Urena B (2001) A model describing the evolution of West Nile-like encephalitis in New York City. Math Comput Model 34:771-781

51. Unnasch R, Sprenger T, Katholi C, Cupp E, Hill G, Unnasch T (2006) A dynamic transmission model of eastern equine encephalitis virus. Ecol Modell 192:425-440

52. van den Driessche P, Watmough J (2002) Reproduction numbers and sub-threshold endemic equilibria for compartmental models of disease transmission. Math Biosci 180:2948

53. Wan H, Zhu H (2010) The backward bifurcation in compartmental models for West Nile virus. Math Biosci 227:20-28

54. Whittle P (1955) The outcome of a stochastic epidemic: a note on Bailey's paper. Biometrika 42:116-122

55. Wonham MJ, de Camino-Beck T, Lewis MA (2004) An epidemiological model for West Nile virus: invasion analysis and control applications. Proc R Soc Lond B Biol Sci 271:501507

56. Wonham MJ, Lewis MA (2008) A Comparative Analysis of Models for West Nile Virus. In: Brauer F, van den Driessche P, Wu J (eds) Mathematical Epidemiology. Lecture Notes in Mathematics, vol 1945. Springer, Berlin, Heidelberg, pp 365-390 